\title{
Estrutura físico-funcional de restaurantes populares do estado do Rio de Janeiro: influência sobre as condições higiênico-sanitárias
}

\section{Physical and functional structure of popular restaurants in the state of Rio de Janeiro: influence on sanitary conditions}

Aline Gomes Mello?

Gizene Luciana Pereira Sales ${ }^{2}$

Lucia Maria Jaeger ${ }^{3}$

Luciléia Granhen Tavares Colares ${ }^{4}$

${ }^{1}$ Nutricionista, professora do Curso de Nutrição da Universidade Federal do Rio de Janeiro, Campus Macaé. Macaé, RJ, Brasil

${ }^{2}$ Nutricionista, professora do Curso de Nutrição da Universidade Potiguar - Escola de Saúde. Natal, RN, Brasil.

${ }^{3}$ Doutorado em Fitotecnia, professora do Curso de Farmácia da Universidade Federal do Rio de Janeiro.

${ }^{4}$ Nutricionista, professora do Curso de Nutrição da Universidade Federal do Rio de Janeiro. Rio de Janeiro, RJ, Brasil

Correspondência / Correspondence Aline Gomes Mello

E-mail: alinegmellori@gmail.com

\section{Resumo}

Os Restaurantes Populares (RPs) têm como objetivo produzir e distribuir refeições saudáveis e em condições higiênico-sanitárias adequadas a preços acessíveis à população em vulnerabilidade social. O presente estudo visou caracterizar os RPs localizados no Estado do Rio de Janeiro, identificando não conformidades que podem influenciar na rotina do processo produtivo das refeições e nas condições higiênico-sanitárias. Verificou-se que as estruturas físicas de $70 \%$ dos RPs foram adaptadas de locais já construídos com outra finalidade como, por exemplo, parte de um estádio de futebol, estação ferroviária e cinema, e que $60 \%$ dos RPs possuem estrutura pavimentar, sendo as refeições produzidas em um pavimento e distribuídas em outro. Segundo a avaliação das condições higiênico-sanitárias da estrutura física, tanto os RPs térreos quanto os pavimentares foram classificados como inadequados, podendo prejudicar a qualidade das refeições servidas e contribuir para os surtos de doenças transmitidas por alimentos, colocando em risco a saúde pública. Os aspectos físico-funcionais devem ser considerados na fase de elaboração do projeto de implantação da unidade de alimentação e nutrição, pois interferem na segurança dos alimentos e podem comprometer o funcionamento correto do serviço de alimentação.

Palavras-chave: Serviços de alimentação. Estrutura dos serviços. Higiene dos alimentos. Boas práticas de manipulação. 


\section{Abstract}

Popular Restaurants (PR's) aim at producing and distributing healthy meals, in adequate sanitary conditions at affordable prices for socially vulnerable people. This study aimed to assess popular restaurants in the State of Rio de Janeiro, identifying nonconformities that may influence the production process routine of meals and sanitary conditions. It was found that the physical structure of $70 \%$ of PR's were adapted from other purpose constructions, for example, part of a football stadium, train station or theater; and 60\% of PR's have flooring structure, the meals being produced on a floor and distributed on another. There was no difference in the adequacy percentage of sanitary conditions among the PR's; all were classified as inadequate. The physical and functional nonconformities may impair the quality of meals served, contributing to outbreaks of foodborne diseases endangering public health. The physical and functional aspects should be considered at the development phase of alimentation and nutrition unit deployment projects, as they interfere with food safety and may compromise the correct operation of the food service.

Key words: Food service. Structure of services. Food hygiene. Good handling practices.

\section{Introdução}

O modo de vida nas médias e grandes cidades tem gerado progressivo crescimento do número de pessoas que realizam suas refeições fora do domicílio. ${ }^{1}$ Esse fato pode ser verificado a partir de dados gerados na Pesquisa de Orçamento Familiar (POF-2008/2009), que mostrou que um terço das refeições dos brasileiros é realizado fora do lar, representando 31\% de sua despesa com alimentação. $^{2}$

A dimensão e a importância do setor de alimentação fora do domicílio na economia nacional podem ser medidas a partir dos números gerados pelo segmento. No ano de 2012, o mercado de refeições coletivas, como um todo, forneceu cerca de 18 milhões de refeições/dia, ofereceu 190 mil empregos diretos e consumiu diariamente um volume de 11 mil toneladas de alimentos. ${ }^{3}$ 
Os Restaurantes Populares (RPs) fazem parte da Política Nacional de Segurança Alimentar e são Unidades de Alimentação e Nutrição (UANs) que têm como princípios fundamentais a produção e a distribuição de refeições saudáveis, em condições higiênico-sanitárias adequadas a preços acessíveis à população em vulnerabilidade social, devendo ser localizados, preferencialmente, em cidades com mais de 100 mil habitantes. ${ }^{4}$ São grandes unidades de produção e comercialização de refeições, administradas pelo poder público, com o objetivo de ampliar a oferta de alimentação saudável e nutricionalmente balanceada, elaborada com produtos regionais, a preços acessíveis, e servidas em locais apropriados e confortáveis, de forma a garantir a dignidade do ato de se alimentar. ${ }^{5}$

Pesquisa desenvolvida pelo Instituto Pólis, em 2005, identificou 111 RPs localizados em 18 estados e 59 municípios, $50 \%$ deles concentrados na Região Sudeste. ${ }^{6}$ No Estado do Rio de Janeiro, os RPs fazem parte da política estadual de segurança alimentar, e atualmente 16 estão em funcionamento, sendo nove na cidade do Rio de Janeiro e um em cada um dos seguintes municípios: Barra Mansa, Campos dos Goytacazes, Duque de Caxias, Itaboraí, Niterói, Resende e Volta Redonda. ${ }^{7}$ Juntos, esses RPs servem, de segunda a sexta-feira, 19.875 cafés da manhã e 51.325 almoços por dia, ao preço de $\mathrm{R} \$ 0,35$ e $\mathrm{R} \$ 1,00$, respectivamente. ${ }^{7}$ Isso enfatiza a importância do desenvolvimento de pesquisas em prol de melhorias desse serviço prestado à população.

Dos 16 RPs localizados no Estado do Rio de Janeiro, cinco receberam apoio do Governo Federal, por meio do Ministério de Desenvolvimento Social e Combate à Fome (MDS), para financiamento de construção, reforma e adaptação de instalações prediais, aquisição de equipamentos permanentes, móveis e utensílios novos e capacitação e formação profissional na área de alimentação e nutrição. ${ }^{5,7}$

A gestão dos RPs é realizada por empresa terceirizada escolhida a partir de processo de licitação, sendo transferida à iniciativa privada a exploração comercial do restaurante, e cabendo à administração pública o subsídio para a oferta das refeições a preços acessíveis, a avaliação e o monitoramento dos serviços prestados pela concessionária. ${ }^{1} \mathrm{O}$ planejamento físico-funcional de qualquer UAN é primordial para a obtenção de instalações adequadas aos objetivos propostos. É a partir desse planejamento que se pode garantir a operacionalização das refeições conforme os padrões qualitativos desejados, do ponto de vista técnico e higiênico-sanitário. ${ }^{8}$

Levando em consideração a relevância dos RPs como política pública e a interferência dos aspectos estruturais no processo de produção de refeições, o presente estudo visou caracterizar a estrutura físico-funcional e avaliar as instalações dos RPs localizados no Estado do Rio de Janeiro, identificando não conformidades que possam influenciar na rotina do processo produtivo das refeições e, consequentemente, nas condições higiênico-sanitárias. 


\section{Materiais e métodos}

Trata-se de estudo transversal e exploratório, realizado no ano de 2007 em dez restaurantes populares localizados no Estado do Rio de Janeiro, um em cada dos seguintes municípios: Duque de Caxias, Niterói, Nova Iguaçu, Itaboraí, Campos dos Goytacazes e Barra Mansa, e quatro no município do Rio de Janeiro. Dos RPs estudados, um (RP Bonsucesso) recebeu apoio financeiro do Ministério de Desenvolvimento Social e Combate à Fome, para reforma de estrutura físicofuncional. O estudo foi aprovado pelo Comitê de Ética em Pesquisa do Instituto de Estudos em Saúde Coletiva da Universidade Federal do Rio de Janeiro (Parecer no 03/2007).

Para a caracterização da estrutura físico-funcional, foi realizada observação sistemática, que segundo Tobar \& Yalour ${ }^{9}$ tem implícita ou explicitamente suposições básicas ou hipóteses, que impulsionam o pesquisador a observar certos fenômenos em detrimento de outros. Além disso, foi elaborado questionário semiestruturado e aplicado por entrevistador treinado aos responsáveis técnicos dos RPs, com questões abertas e fechadas sobre o tipo de serviço prestado e contrato, número de refeições produzidas, tipo e composição do cardápio, sistema de distribuição das refeições, horário de funcionamento do restaurante, bem como a composição das preparações. Procurou-se identificar as peculiaridades que podem exercer influência direta no processo produtivo de refeições e nas condições higiênico-sanitárias.

$\mathrm{Na}$ avaliação das instalações dos RPs, foram observados aspectos relacionados a localização, zoneamento, setorização e ambiente, tomando por base o roteiro de implantação de restaurantes populares publicado pelo Ministério de Desenvolvimento Social e Combate a Fome. ${ }^{4}$ Para avaliação das condições higiênico-sanitárias, utilizou-se o roteiro de inspeção sanitária (RIS) elaborado com base na Resolução RDC n. 216/2004 da Agência Nacional de Vigilância Sanitária, composto de 103 itens distribuídos em 12 blocos: I) Armazenamento dos alimentos; II) Estrutura física; III) Equipamentos e utensílios; IV) Higiene ambiental; V) Manejo de resíduos sólidos; VI) Manipuladores de alimentos; VII) Preparo de alimentos; VIII) Distribuição; IX) Controle integrado de vetores e pragas urbanas; X) Abastecimento de água; XI) Documentação; e XII) Capacitação dos manipuladores de alimentos. ${ }^{10}$

O percentual de adequação das condições higiênico-sanitárias (PACHS) de cada RP foi baseado nos pontos de corte sugeridos na Resolução RDC n. 275/2002, considerando adequado o estabelecimento com PACHS superior ou igual a 76\%; parcialmente adequado, entre 51 e $76 \%$; e inadequado, inferior ou igual a $50 \% .^{11}$ 


\section{Resultados e discussão}

\section{Caracterização dos Restaurantes Populares}

Os RPs estudados possuem características semelhantes: funcionam de segunda a sexta-feira e distribuem desjejum das $6 \mathrm{~h}$ às $9 \mathrm{~h}$ e almoço das $10 \mathrm{~h}$ às $15 \mathrm{~h}$. Servem juntos cerca de 12.000 desjejuns e 30.000 almoços diariamente.

A operacionalização dos RPs se faz a partir da prestação de serviço terceirizado de fornecimento de refeições, por meio de contrato firmado entre a Secretaria de Estado de Assistência Social e Direitos Humanos (SEASDH) e a empresa especializada em alimentação para coletividade, com base no processo licitatório para prestação de serviço de preparo e fornecimento de alimentação. ${ }^{7}$ O contrato é do tipo gestão a preço fixo, no qual a contratada é responsável pelo desenvolvimento de todas as etapas do processo de produção das refeições e todos os custos e gastos administrativos e operacionais, sendo paga por unidades produzidas. ${ }^{12}$

O cardápio é do tipo popular, composto de preparações simples e de baixo custo, incluindo: sopa, salada, arroz, feijão, prato principal, opção de prato principal, guarnição, sobremesa, refresco, pão, cafezinho ou chá. A distribuição da refeição é feita por cafeteria fixa, e as preparações são servidas aos comensais por manipuladores de alimentos. ${ }^{13}$

Com relação ao público beneficiário, os RPs estudados têm peculiaridades, de acordo com a localização, sendo compostos por trabalhadores formais e informais de baixa renda, desempregados, estudantes, aposentados, moradores de rua e famílias em situação de risco de insegurança alimentar e nutricional. ${ }^{4}$ Quanto à estrutura física, 70\% dos RPs estudados foram adaptados de locais já construídos com outra finalidade como, por exemplo, parte de um estádio de futebol, estação ferroviária e cinema; $60 \%$ dos RPs possuem estrutura pavimentar, sendo as refeições produzidas em um pavimento e distribuídas em outro (quadro 1).

Observou-se comprometimento do processo produtivo das refeições nos RPs pavimentares, principalmente quando havia problema no monta-carga. Isso acarretava sobrecarga de trabalho dos manipuladores de alimentos e aumento do tempo de espera dos comensais, comprometendo a qualidade das refeições servidas. 
Quadro 1. Caracterização dos RPs do Estado do Rio de Janeiro quanto ao número de pavimentos, mês e ano de inauguração e tipo de estrutura física. Rio de Janeiro-RJ, 2007.

\begin{tabular}{|c|c|c|c|}
\hline Municípios & $\begin{array}{c}\text { No de } \\
\text { Pavimentos }\end{array}$ & $\begin{array}{l}\text { Mês/Ano de } \\
\text { Inauguração }\end{array}$ & $\begin{array}{l}\text { Tipo de } \\
\text { estrutura }\end{array}$ \\
\hline \multicolumn{4}{|l|}{ Rio de Janeiro/Bairros } \\
\hline Bangu & 02 & $12 / 2001$ & \multirow{7}{*}{ Adaptada } \\
\hline Bonsucesso & 02 & $07 / 2007$ & \\
\hline Centro & 02 & $11 / 2000$ & \\
\hline Maracanã & 01 & 04/2002 & \\
\hline Duque de Caxias & 01 & $12 / 2001$ & \\
\hline Itaboraí & 02 & 04/2002 & \\
\hline Niterói & 02 & $02 / 2002$ & \\
\hline Barra Mansa & 02 & $08 / 2005$ & \multirow{3}{*}{ Não adaptada } \\
\hline Campos dos Goytacases & 01 & $09 / 2004$ & \\
\hline Nova Iguaçu & 01 & 04/2002 & \\
\hline
\end{tabular}

Embora apresentem melhor operacionalização do serviço, os RPs com estrutura linear também sofrem influência das adaptações estruturais, que comprometem o fluxo de materiais, pessoas e preparações. Vale ressaltar que normas técnicas de edificações e ergonomia devem ser consideradas na construção ou reforma das áreas de trabalho em UANs, para que haja melhor funcionamento da unidade e menor desconforto para os manipuladores, como apontam Aguiar et al.. ${ }^{14}$

\section{Instalações dos Restaurantes Populares}

Ao comparar as instalações dos RPs com o proposto no roteiro de implantação de restaurantes populares, ${ }^{4}$ foram observadas inadequações somente naqueles que não receberam apoio do MDS (tabela 1). Vale ressaltar que no roteiro do MDS constam recomendações baseadas na legislação vigente, que deve ser seguida na concepção e implantação de serviços de alimentação. 
Tabela 1. Comparativo entre as instalações dos nove RPs do Estado do Rio de Janeiro que não receberam apoio do MDS e o proposto no Roteiro de Implantação de Restaurantes Populares. Rio de Janeiro-RJ, 2007.

\begin{tabular}{ccc}
$\begin{array}{c}\text { Itens constantes do Roteiro de } \\
\text { Implantação de RP* }\end{array}$ & $\begin{array}{c}\text { Número de } \\
\text { RPs* }\end{array}$ & $\begin{array}{c}\text { Adequação } \\
\%\end{array}$ \\
\hline Localização & 09 & 100 \\
Zoneamento & 02 & 22 \\
Setorização & 01 & 11 \\
Ambientes & 01 & 11 \\
\hline
\end{tabular}

*Restaurantes Populares

Todos os RPs estão localizados em regiões com grande circulação de pessoas, sendo de fácil acesso ao comensal, evitando assim a utilização de transporte para seu deslocamento durante o horário do almoço. Quanto ao zoneamento, 88\% dos RPs estão expostos a inundações, poeira e odores indesejáveis, por conta das inadequações estruturais encontradas e até mesmo pela localização geográfica dos grandes centros urbanos (tabela 1).

Com relação à setorização necessária para o funcionamento adequado dos estabelecimentos que manipulam alimentos, as áreas destinadas a setores como recepção/pré-higienização da matériaprima, armazenamento dos gêneros, pré-preparo, cocção, distribuição-refeitório, higienização dos utensílios, depósito de resíduos e setores complementares ou eventuais devem ser bem definidas e são imprescindíveis para viabilizar as tarefas exigidas no processo produtivo das refeições, conforme sugerido pela Resolução RDC n. 216/2004.10

A etapa de recepção e controle de matéria-prima deve ser realizada em área externa, coberta e dotada de plataforma para descarga de gêneros alimentícios. ${ }^{10}$ Foi observado que nos nove RPs que receberam apoio do MDS não existe área específica para esse fim, e a matéria-prima é levada diretamente para a área de estocagem, sem prévia inspeção. Essa inadequação foi também observada por Correia \& Rocha, ${ }^{15}$ que estudaram 20 serviços de alimentação localizados em Portugal, o que aumenta a possibilidade de armazenamento de gêneros impróprios para consumo. 
A inadequação do dimensionamento de área e equipamentos e da setorização pode gerar obstáculos na organização do trabalho e favorecer o fluxo cruzado entre gêneros alimentícios in natura e processados, bem como pessoas e resíduos sólidos, consequentemente aumentando o risco de contaminação das preparações e interferindo de forma negativa na qualidade das refeições, fato observado nos RPs estudados e por outros autores. ${ }^{15,16}$

Quanto aos ambientes de trabalho, foram observadas as seguintes inadequações: pisos molhados e desconforto térmico, especialmente nas áreas de produção, higienização mecânica e manual. Essas não conformidades ambientais também foram relatadas por diversos autores, como Colares \& Freitas, ${ }^{17}$ Glina et al., ${ }^{16}$ Aguiar et al.${ }^{14}$ e Correia \& Rocha,${ }^{15}$ que relacionam as condições de trabalho inadequadas ao desenvolvimento de agravos à saúde dos trabalhadores, reduzindo a capacidade produtiva dos mesmos. Portanto, medidas preventivas devem ser adotadas durante a concepção e implantação dos serviços de alimentação.

\section{Condições higiênico-sanitárias dos Restaurantes Populares}

Ao avaliar as condições higiênico-sanitárias dos RPs estudados, podem-se observar inadequações, independentemente de sua estrutura (pavimentar ou linear), sendo todos classificados como parcialmente adequados (PACHS entre 51 e 76\%) (tabela 2).

Com relação à estrutura física, foram avaliados aspectos referentes à edificação, como: estado de conservação do piso, parede, teto e portas; instalação sanitária dos funcionários e comensais; fluxo de ar artificial e iluminação. Verificou-se que todos os RPs (lineares e pavimentares) foram classificados como inadequados (tabela 2). Esse resultado mostra que a estrutura física pode contribuir de forma negativa para as condições higiênico-sanitárias dos estabelecimentos.

Tabela 2. Adequação das condições higiênico-sanitárias dos RPs em relação ao total de blocos avaliados e à estrutura física. Rio de Janeiro-RJ, 2007.

\begin{tabular}{ccc}
\hline & \multicolumn{2}{c}{ Percentual de adequação higiênico-sanitária } \\
\cline { 2 - 3 } Estrutura & PACHS* & PACHS* \\
& Total & Estrutura física \\
\hline Linear $(\mathrm{n}=4)$ & $55 \%$ & $42 \%$ \\
Pavimentar $(\mathrm{n}=6)$ & $62 \%$ & $50 \%$ \\
\hline
\end{tabular}

*Percentual de Adequação das Condições Higiênico-Sanitárias: Adequado - PACHS $\geq 76 \%$; Parcialmente adequado - PACHS entre 51 e $76 \%$; e Inadequado - PACHS $\leq 50 \%$. 
Os RPs estudados foram inaugurados entre os anos 2000 e 2007 (quadro 1). Embora tenham construção relativamente nova, as principais não conformidades encontradas estavam relacionadas à setorização (falta de acesso exclusivo de materiais às instalações dos restaurantes) e à má conservação da estrutura física (paredes e tetos com infiltração e vazamentos; pisos com rachaduras; portas de madeira descascadas e estufadas). E apesar de a conservação e a manutenção predial e dos equipamentos adquiridos pelo governo do estado serem de responsabilidade da empresa contratada, cabe ao estado vistoriar, contabilizar, patrimoniar e fiscalizar a conservação dos bens - portanto, contratada e contratante devem zelar pelo bom funcionamento da estrutura físico-funcional da UAN. Essas não conformidades vêm sendo relatadas em serviços de alimentação de diversos ramos, e a manutenção predial e de equipamentos tem sido apontada como fator importante na produção de refeições seguras do ponto de vista higiênico-sanitário. ${ }^{15,16,18,19}$

Em 50\% dos RPs, as instalações sanitárias dos funcionários e o armazenamento temporário de resíduos estavam localizados próximos à área de recebimento e armazenamento de gêneros, evidenciando as adaptações realizadas na estrutura física. Essas inadequações podem comprometer a qualidade das refeições, aumentando o risco de contaminação, como apontado em estudo realizado por Mendonça et al.. ${ }^{20}$

Os aspectos físico-funcionais devem ser considerados na fase de elaboração do projeto de implantação da UAN, pois interferem na segurança dos alimentos e podem comprometer o funcionamento correto do serviço de alimentação.

\section{Conclusões}

Nos RPs estudados, há dissonância entre o preconizado pela legislação sanitária e o manual de implantação de restaurantes populares e o observado - ou seja, o processo operacional se torna inadequado diante de adaptações estruturais que buscam atender à funcionalidade dos serviços, influenciando diretamente na execução da rotina de atividades.

Independentemente do tipo de estrutura (pavimentar ou linear), os restaurantes populares apresentaram inadequações físico-funcionais que podem comprometer as condições higiênicosanitárias, tendo em vista que os mesmos foram classificados como parcialmente adequados e inadequados especificamente em relação à estrutura física.

Recomenda-se investimento para reestruturação e modernização dos RPs em questão, buscando atender, de fato, ao objetivo proposto pelo Programa Restaurante Popular, visando oferecer à população refeições saudáveis e atender à legislação vigente em relação ao funcionamento dessas unidades. Destaca-se a importância do envolvimento do governo e da empresa terceirizada responsável na busca de melhorias no serviço prestado à população. 


\section{Referências}

1. Brasil. Ministério do Desenvolvimento Social e Combate à Fome. Restaurante Popular: Apresentação do Programa. Brasília: Ministério do Desenvolvimento Social e Combate à Fome; 2007. 11 p.

2. IBGE. Coordenação de Trabalho e Rendimento. Pesquisa de Orçamentos Familiares 2008-2009: despesas, rendimentos e condições de vida. Rio de Janeiro: IBGE; 2010. 222 p.

3. Associação Brasileira das Empresas de Refeições Coletivas [bomepage na internet]. Mercado real [acesso em 2012 nov 6]. Disponível em: http://www.aberc.com.br/base.asp?id=2.

4. Brasil. Ministério do Desenvolvimento Social e Combate à Fome [homepage na internet]. Restaurantes populares: roteiro de implantação; 2007. [acesso em 2012 jun 14]. Disponível em: http://www.mds. gov.br/segurancaalimentar/equipamentos/restaurantespopulares/comoimplantar/?searchterm=rest aurantes $\% 20$ populares $\% 20$ roteiro $\% 20 \mathrm{de} \% 20$ implanta $\% \mathrm{C} 3 \% \mathrm{~A} 7 \% \mathrm{C} 3 \% \mathrm{~A} 3 \mathrm{O}$.

5. Brasil. Ministério do Desenvolvimento Social e Combate à Fome [homepage na internet]. Restaurante Popular [acesso em 2012 jun 14]. Disponível em: http://www.mds.gov.br/segurancaalimentar/ equipamentos/restaurantespopulares

6. Pólis-Instituto de Estudos, Formação e Assessoria em Políticas Sociais. Síntese produzida a partir do relatório analítico da pesquisa, entregue ao Ministério do Desenvolvimento Social em junho de 2005. [acesso em 2012 jul 17]. Disponível em: http://www.polis.org.br/download/174.pdf.

7. Rio de Janeiro (Estado). Secretaria de Assistência Social e Direitos Humanos. Restaurante Cidadão. [acesso em: 2012 nov 14]. Disponível em: http://www.rj.gov.br/web/seasdh/exibeconteudo?articleid $=922982$

8. Teixeira SMFG, Milet Z, Carvalho J, Biscontini TM. Administração aplicada às unidades de alimentação e nutrição. São Paulo: Atheneu; 2010. 230 p.

9. Tobar F, Yalour MR. Como fazer teses em saúde pública: conselhos e idéias para formular projetos e redigir teses e informes de pesquisa. Rio de Janeiro: Fiocruz; 2001. 172 p.

10. Agência Nacional de Vigilância Sanitária (Brasil). Resolução RDC n. 216, de 15 de setembro de 2004. Dispõe sobre regulamento técnico de boas práticas para serviços de alimentação. Diário Oficial da União. 2004 set. 16, Seção 1: 25.

11. Agência Nacional de Vigilância Sanitária (Brasil). Resolução RDC nº 275, de 21 de outubro de 2002. Estabelece Procedimentos Operacionais Padronizados que contribuam para a garantia das condições higiênico-sanitárias necessárias ao processamento / industrialização de alimentos, complementando as Boas Práticas de Fabricação. Diário Oficial da União. 2003 out. 23.

12. SENAC. Administração na alimentação coletiva. São Paulo: SENAC/SP; 2007. 129 p.

13. Secretaria Estadual de Assistência Social e Direitos Humanos. Termo de Referência - prestação de serviço de preparo, fornecimento e distribuição de alimentação a preços populares no restaurante popular. Documento interno. 2009. 
14. Aguiar OB, Valente JG, Fonseca MJM. Descrição sócio-demográfica, laboral e de saúde dos trabalhadores do setor de serviços de alimentação dos restaurantes populares do estado do Rio de Janeiro. Rev. de Nutrição. 2010;23(6):969-82.

15. Correia MJFS, Rocha AAMCN. A importância dos fatores estruturais na garantia da segurança alimentar na produção de refeições para escolares. Demetra. 2012;7(1):39-46.

16. Glina DMR, Siqueira AR, Rocha LER. Análise ergonômica da higienização de loucas de um hospital cardiológico público. Mundo saúde 2008;32(2):198-207.

17. Colares LGT, Freitas CM. Processo de trabalho e saúde de trabalhadores de uma unidade de alimentação e nutrição: entre a prescrição e o real do trabalho. Cad saúde pública. 2007;23(12):3011-20.

18. Veiga CF, Doro DL, Oliveira KMP, Bombo DL. Estudo das condições sanitárias dos estabelecimentos comerciais de manipulação de alimentos do município de Maringá, PR. Hig. aliment. 2006;20(138):28-37.

19. Souza CH, Sathler J, Jorge MN, Horst RFMSL. Avaliação das condições higiênico sanitárias em uma unidade de alimentação e nutrição hoteleira, na cidade de Timóteo-Mg. Nutrir gerais. 2009;3(4):312-29.

20. Mendonça SKD, Souza BF, Almeida OS. Avaliação estrutural e higiênicosanitária de duas unidades de alimentação e nutrição em Macapá-Ap. Nut. pauta. 2012:29-33.

Recebido: 01/2/2013

Revisado: 04/4/2013

Aprovado: 22/5/2013 
